\title{
An Improved Artificial Bee Colony Algorithm in LoRa Wireless Communication System for Efficient Multimedia Transmission
}

\author{
Yan Song, ${ }^{1}$ Lidong Huang $\mathbb{D}^{1},{ }^{1}$ Panfeng Xu, ${ }^{1}$ Lili Li, ${ }^{1}$ Min Song, ${ }^{2}$ and Yue Long ${ }^{1}$ \\ ${ }^{1}$ College of Physics, Liaoning University, Shenyang, China \\ ${ }^{2}$ College of Information, Shenyang Institute of Engineering, Shenyang, China \\ Correspondence should be addressed to Lidong Huang; 4031731893@smail.lnu.edu.cn
}

Received 11 September 2018; Revised 28 October 2018; Accepted 18 November 2018; Published 3 December 2018

Guest Editor: Kai Wang

Copyright (c) 2018 Yan Song et al. This is an open access article distributed under the Creative Commons Attribution License, which permits unrestricted use, distribution, and reproduction in any medium, provided the original work is properly cited.

\begin{abstract}
Video streaming communication networks will be a very important way to send multimedia information anytime and anywhere, and the construction of the network base station which transmits signals is crucial in future. However, there is a contradiction between the power consumption of LoRa nodes and the real-timeliness of mesh network. In order to solve the contradiction, this article aims to combine the mesh network of LoRa wireless communication system with an improved artificial bee colony algorithm. Specifically, an artificial bee colony algorithm, which is based on RBF radial basis neural network trained with random gradient method, is designed. Simulation results show that the proposed algorithm solves the contradiction between power consumption and real-timeliness effectively. When using this improved network system structure to send multimedia information, it shows obvious superiority in terms of the high efficiency and real-timeliness of multimedia transmission.
\end{abstract}

\section{Introduction}

With the development of wireless communication network technology, the advantages of intelligent productions of wireless communication are widely accepted, such as low cost and good scalability [1-3]. These productions have been paid more and more attention by the society $[4,5]$. Moreover, the transmission of multimedia information is getting larger and more global. Video streaming and other high-capacity media transmission technologies require better wireless networks architecture $[5,6]$.

The LoRa technology solves the problem of long distance and low power consumption successfully. Compared to other wireless transmission technologies, LoRa has the advantages of longer transmission distance and lower consumption [57]. However, the existing wireless transmission technology cannot meet the real-time monitoring effect of wireless network under the conditions of low power consumption and long distance. In the existing methods, there is a mesh network architecture that minimizes the number of devices, which greatly reduces the cost of base station establishment $[8,9]$. At the same time, it is very convenient to deploy the equipment [10-12]. The mesh network architecture is very stable, and it is not affected by a single node. When a near node fails or is disturbed, the packet will be transferred automatically to the alternate path for transmission. Moreover, it has flexible structure and advantages in terms of overloading and communication load balancing [13-15]. This kind of mesh network reduces the interference of the adjacent user wireless network when transmitting data, and its efficiency of information transmission has been improved greatly.

To a certain extent, the mesh network reduces the power consumption and achieves the coverage of a larger area, but it cannot completely solve the contradiction between the power consumption and real time of LoRa wireless network [1619]. Some additional interference or space extension may result in loss of signal transmission capability. We consider adopting the basic artificial swarm algorithm. There is still a problem that the wireless network layout may be optimized correspondingly [20,21], but the effect of optimization is not obvious enough and it converges slowly. When looking for the optimal solution in space, there may be a deviation. Moreover, the traditional method in the algorithm will affect the convergence speed of the optimal solution greatly. Therefore, this 
existing method cannot deal with the contradiction between power consumption and real time $[11,16,22]$, which is an obstacle to the multimedia information transmission. To fill this gap, the paper proposes an improved algorithm to solve the problem.

The contributions of this paper are listed as follows: an artificial bee colony algorithm based on RBF radical basis neural network (RBFABC) is designed to resolve this problem, and this algorithm can solve the contradiction between power consumption and real-timeliness [14, 17, 23]. Furthermore, this design not only improves the traditional way of selecting and updating the honey sources in basic artificial swarm optimization algorithm, but also enables the algorithm to converge towards the optimal solution. Moreover, the convergence speed of the proposed algorithm is much higher. The improved algorithm not only ensures the real-time performance of wireless network $[14,16,24]$, but also reduces the power consumption of wireless network. In brief, the improved algorithm overcomes technical obstacles in the field of multimedia wireless network, and it has great significance to the development of wireless transmission technology.

The rest of this paper is organized as follows: Section 2 introduces the basic artificial swarm algorithm. Section 3 presents the system model. Section 4 proposes the RBFABC algorithm. Section 5 performs simulations to verify the effectiveness of the proposed algorithm. Section 6 concludes this paper.

\section{Basic Artificial Swarm Algorithm}

As everyone knows, bees have special behavioral patterns for finding honey sources $[17,18]$. In general, the process of searching for honey sources is divided into three parts: looking for the honey sources; updating the honey sources; selecting the best honey sources $[14,25,26]$. The algorithm flow is shown in Algorithm 1.

In this algorithm, the leader bees have a memory function, which can save the information of the honey sources. Moreover, the leader bees share the honey sources information with orientation bees. According to their dance of the leader bees, the orientation bees determine which direction to go. The observation bees are responsible for searching nectar around the hive, and the leader bees search for honey sources $[27,28]$. We define some variables to represent them $[16,17$, 23]. Firstly, a series of original honey sources are initialized randomly. $\boldsymbol{X}=\left(X_{1}, X_{2}, X_{3}, \ldots, X_{n}\right)$ represents $n$ honey sources $[18,19,25]$. The position of the $m$-th honey source is $\boldsymbol{X}_{\boldsymbol{m}}=\left[X_{m 1}, X_{m 2}, X_{m 3}, \ldots, X_{m n}\right](m=1,2,3, \ldots, n)$, which are potential solutions to the problem $[26,27]$. Then the fitness of honey sources are evaluated by the following formula:

$$
\text { fit }_{m}= \begin{cases}\frac{1}{1+f_{m}} & f_{m}>0 \\ 1+\left|f_{m}\right| & f_{m}<0\end{cases}
$$

where $f_{m}$ represents the target function value of $m$-th solutions. After that, the location of $n$ honey sources updates in a certain way. The process is described as follows.

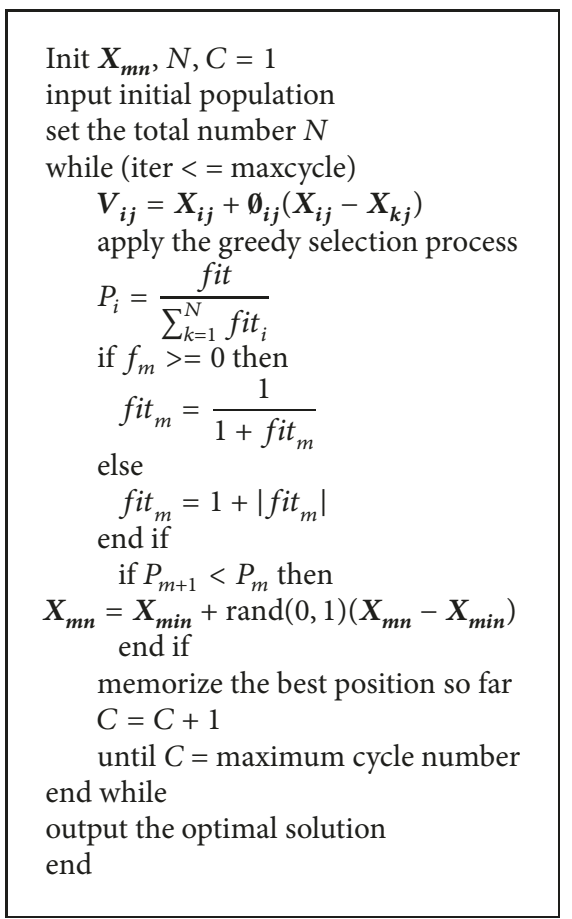

Algorithm 1: The program diagram of the artificial swarm optimization algorithm.

$$
\boldsymbol{X}_{m}^{n}=\boldsymbol{X}_{\text {min }}^{n}+\operatorname{rand}(0,1)\left(\boldsymbol{X}_{\text {max }}^{n}-\boldsymbol{X}_{\text {min }}^{n}\right)
$$

If the honey sources are better, we will select the better one instead of the old one; otherwise keep the old one. If all the leader bees finished searching, the honey sources information will be transmitted to the observation bees $[4,13]$. Then the observation bees choose leader bees according to the fitness value $[28,29]$, and the selection probability is described as follows:

$$
\begin{aligned}
P_{m} & =\frac{f i t_{m}}{\sum_{i=1}^{n} f i t_{m}} \\
\boldsymbol{U}_{\boldsymbol{i j}} & =\boldsymbol{X}_{\boldsymbol{i j}}+\boldsymbol{r}_{\boldsymbol{i j}}\left(\boldsymbol{X}_{\boldsymbol{i} \boldsymbol{j}}-\boldsymbol{X}_{\boldsymbol{k} \boldsymbol{j}}\right)
\end{aligned}
$$

where $\boldsymbol{X}_{\boldsymbol{i}}$ represents the location of honey sources, $\boldsymbol{U}_{\boldsymbol{i}}$ represents the nearby location of $\boldsymbol{X}_{\boldsymbol{i} \boldsymbol{j}}, \boldsymbol{r}_{\boldsymbol{i} \boldsymbol{j}}$ represents the random number within $(-1,1), P_{m}$ represents the selective probability, and fit $_{m}$ represents the fitness of honey sources. If the searching process is over, the honey sources information will be transmitted to the leader bees. Then the leader bees choose a direction according to fitness of honey sources. If this honey source is not replaced after finite loops, it will be abandoned. Finally the leader bees will look for the new honey sources.

\section{System Model}

In order to achieve the effect of algorithm optimization, we analyze the parameters of network architecture and establish the mathematical model of wireless network. As shown in Figure 1. 


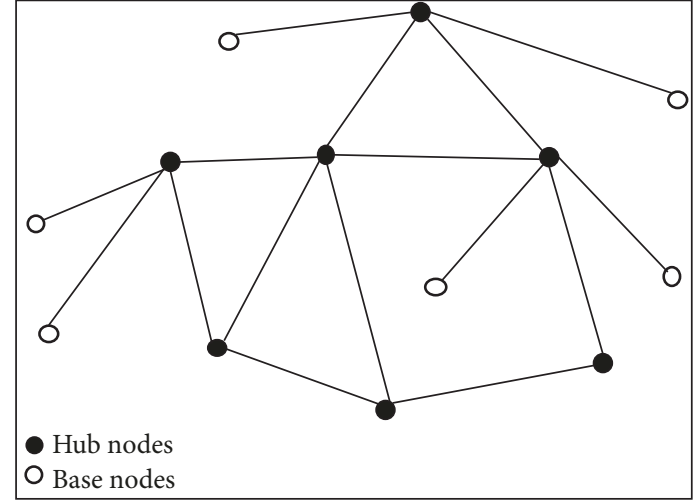

FIGURE 1: Mesh network model.

Beyond that, this paper combines the central function with the improved algorithm. In wireless mesh networks, a small amount of wireless devices can be required to cover a large range $[8,11,15]$. In other words, it is very convenient to create a mathematical model of wireless network [16-18]. The correlation signal transmission capability function of network nodes created is described as follows.

$$
\begin{aligned}
& c_{m}=w_{1} \alpha_{m 1}+w_{2} \alpha_{m 2}+w_{3} \alpha_{m 3}+w_{4} \alpha_{m 4}+w_{5} \alpha_{m 5} \\
& w_{1}+w_{2}+w_{3}+w_{4}+w_{5}=1
\end{aligned}
$$

The signal coverage range of nodes are related to the performance of the node $\left(\alpha_{m 1}\right)$, the location of deployment $\left(\alpha_{m 2}\right)$, the signal coverage $\left(\alpha_{m 3}\right)$, the band width $\left(\alpha_{\mathrm{m} 4}\right)$, and the anti-interference capability $\left(\alpha_{m 5}\right)$. The above factors have different effects on the signal coverage capability of nodes, and $w$ is used to reflect the degree of influence of factors [7]. Under ideal conditions, the relationship between the transmitting power consumption and propagation distance of nodes is described as follows:

$$
P=s+k \lg d+k \lg f
$$

where $P$ represents transmission power, $d$ represents coverage range, $f$ represents transmission frequency, $s$ represents initial effective distance, and $k$ represents effective radiofrequency factor $[7,16,18]$. In order to balance the relationship between power consumption and real time, the mathematical model is established as follows:

$$
\begin{aligned}
T_{m} & =\frac{C_{m}}{C_{\max }} \\
P & =s+k \lg d T_{m}+k \lg f
\end{aligned}
$$

where $T_{m}$ represents the signal coverage capability after quantization, $C_{m}$ represents the signal coverage capability of nodes, and $C_{\max }$ represents the maximum ability of nodes signal coverage.

\section{RBFABC Algorithm}

Although the artificial bee colony algorithm works well, there is still a problem of slow convergence rate in solving the problem of nodes optimization. At the same time, the choice of roulette in the original algorithm may make the algorithm fall into the local optimum. Therefore, in each iteration, there will be an error with the newly generated solution due to each local optimum. Because of the limitation of adaptive value, the speed of the algorithm convergence is affected. The gradient method, which is in the RBF radial basis neural network algorithm, is used to deal with the updating factors $[10,21,25,26]$. Thus we improve the updating factors of honey sources by the RBF. Firstly, within a specified range, a series of original nodes locations are initialized randomly. $\boldsymbol{X}=\left(X_{1}, X_{2}, X_{3}, \ldots, X_{n}\right)$ represents $n$ honey sources. The position of the $m$-th honey source is described as follows.

$$
\boldsymbol{X}_{\boldsymbol{m}}=\left[X_{m 1}, X_{m 2}, X_{m 3}, \ldots, X_{m n}\right]
$$

This is a potential solution to the optimization process $[9,11]$. The degree of honey source is estimated by the instantaneous error function [24-27], as follows:

$$
\begin{aligned}
J(n) & =\frac{1}{2}|\boldsymbol{e}(\boldsymbol{n})|^{2} \\
& =\frac{1}{2}\left|y_{d}-\sum_{k=1}^{N} \boldsymbol{w}_{\boldsymbol{k}}(\boldsymbol{n}) \emptyset\left\{\boldsymbol{x}(\boldsymbol{n}), \boldsymbol{c}_{\boldsymbol{k}}(\boldsymbol{n})\right\}\right|^{2}
\end{aligned}
$$

where $\boldsymbol{w}(\boldsymbol{n})$ represents the weighted value of the impact factors, $\boldsymbol{c}_{\boldsymbol{k}}(\boldsymbol{n})$ represents the center of the radial basis function, and $\boldsymbol{\delta}_{\boldsymbol{k}}(\boldsymbol{n})$ represents variance $[27,28]$.

When the instantaneous error is less than the specified error, the current honey sources are selected as the target honey sources. Moreover, the formula of searching for honey sources is described as follows.

$$
\begin{aligned}
& \boldsymbol{y}_{\boldsymbol{m}}=\sum_{k=1}^{N} \boldsymbol{w}_{\boldsymbol{m} k}\left[s+k \lg \left\|\boldsymbol{x}(\boldsymbol{n})-\boldsymbol{c}_{\boldsymbol{k}}(\boldsymbol{n})\right\|^{2}+k \lg f\right] \\
& \boldsymbol{w}(\boldsymbol{n}+\mathbf{1})=\boldsymbol{w}(\boldsymbol{n})-\mu_{w} \frac{\partial}{\partial \boldsymbol{w}} J(n) \\
& \boldsymbol{c}_{\boldsymbol{k}}(\boldsymbol{n}+\mathbf{1})=\boldsymbol{c}_{\boldsymbol{k}}(\boldsymbol{n})-\mu_{c} \frac{\partial}{\partial \boldsymbol{c}_{\boldsymbol{k}}} J(n) \\
& \boldsymbol{\delta}_{\boldsymbol{k}}(\boldsymbol{n}+\mathbf{1})=\boldsymbol{\delta}_{\boldsymbol{k}}(\boldsymbol{n})-\mu_{\delta} \frac{\partial}{\partial \boldsymbol{\delta}_{\boldsymbol{k}}} J(n)
\end{aligned}
$$

During the updating process $[22,23,26], \boldsymbol{w}(\boldsymbol{n}+\mathbf{1}), \boldsymbol{c}_{\boldsymbol{k}}(\boldsymbol{n}+$ $\mathbf{1})$, and $\boldsymbol{\delta}_{\boldsymbol{k}}(\boldsymbol{n}+\mathbf{1})$ will be updated according to the network correction equation. If these honey sources are not replaced, the position will be reserved. Then the leader bees continue to search for new honey sources. Finally, the new honey sources will be searched as a substitute of position by the improved updating formula. This process is shown in Algorithm 2.

The general steps of the RBFABC algorithm are as follows: initializing a series of original nodes location; searching for the position of some new nodes around the initialized nodes; calculating the instantaneous error value of the position of the new nodes; selecting the better nodes $[6,7,27]$; generating some new nodes around the better nodes by the updating formula; selecting better nodes with instantaneous 


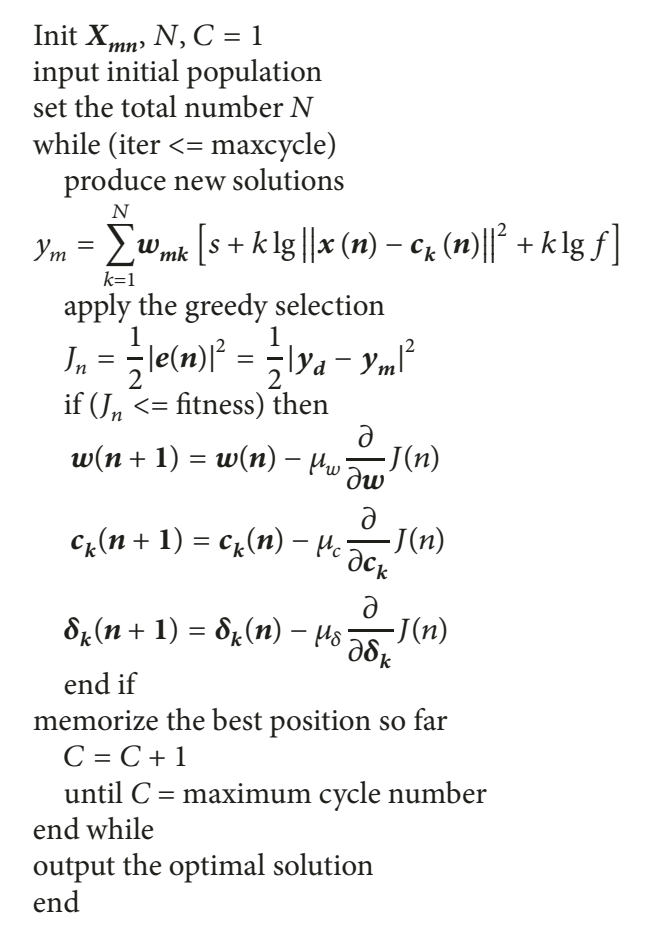

Algorithm 2: Program diagram of the RBFABC algorithm.
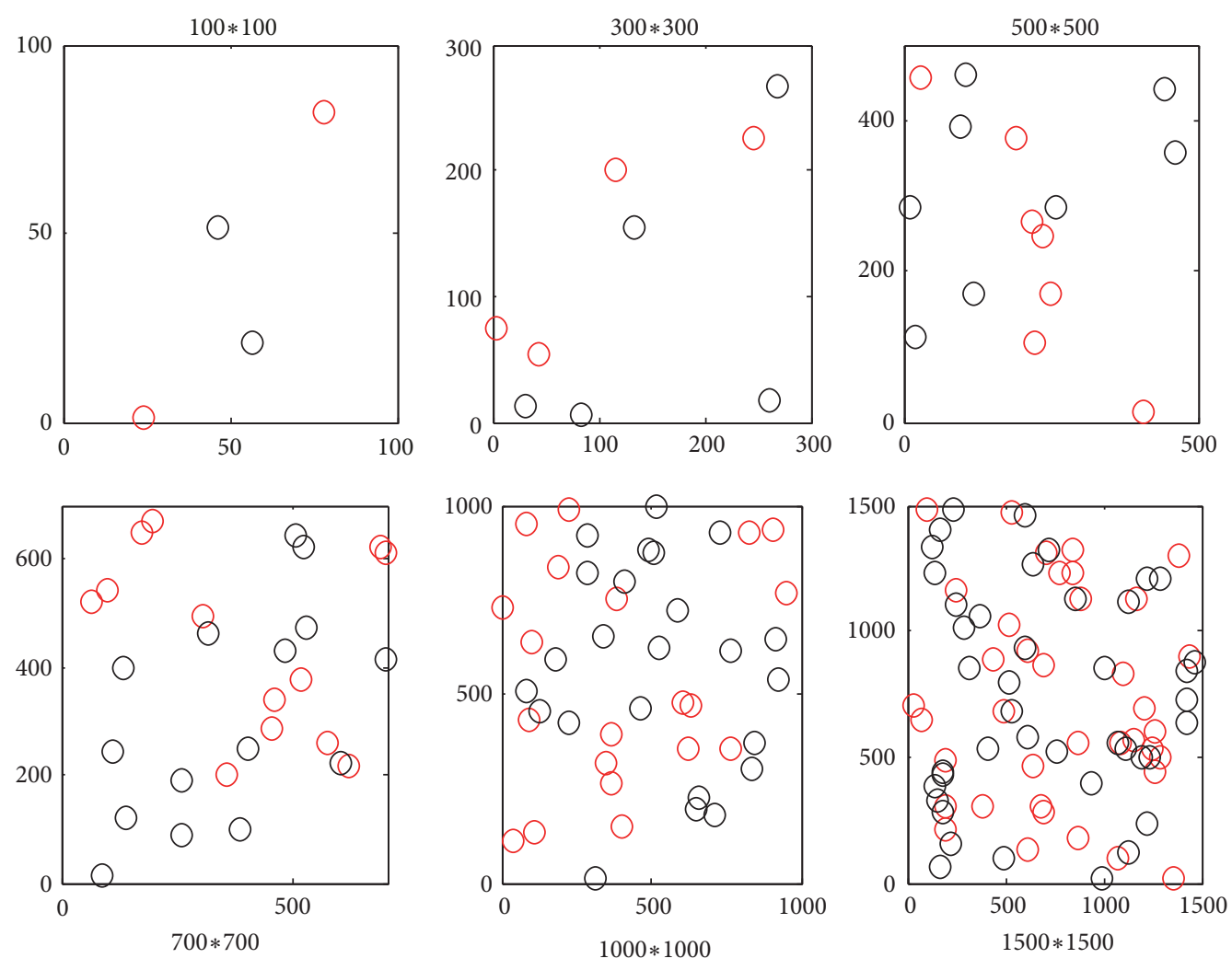
RBFABC
$\mathrm{ABC}$

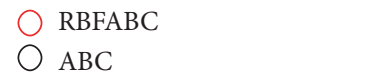

RBFABC

$\mathrm{ABC}$

FIGURE 2: Node distribution under the same area. 


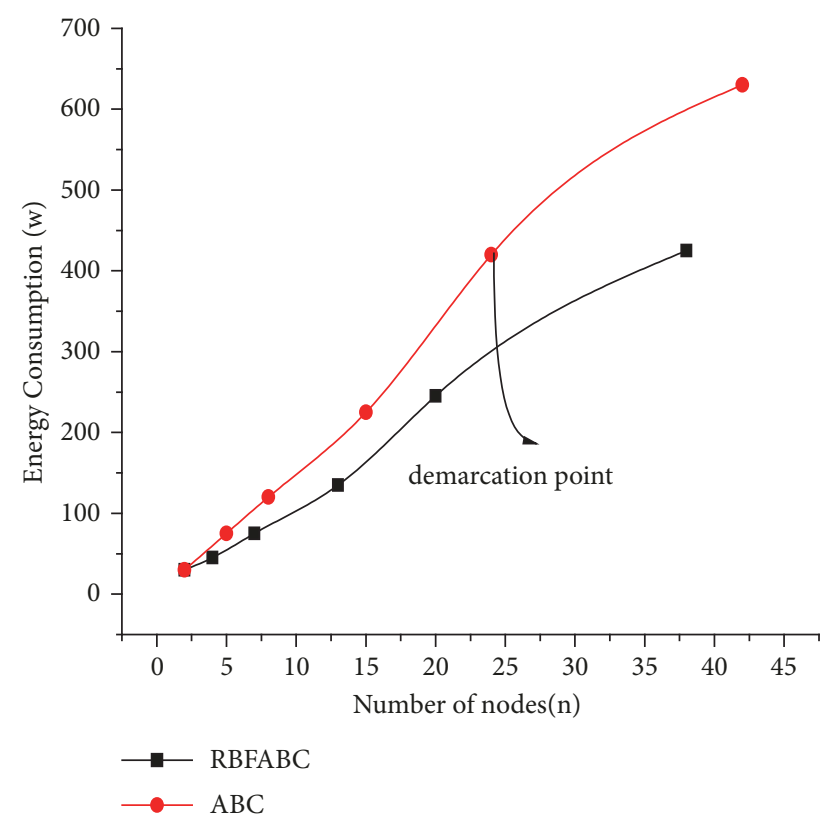

FIGURE 3: Power consumption diagram corresponding to different nodes.

error values; judging whether there are points which need to be abandoned, and if so, these nodes are converted to the alternate nodes; searching for new nodes which are based on the formula $[27,28,30]$; storing the location of optimal nodes so far; evaluating whether the location of nodes meet the stopping condition of optimization, and if so, outputting the most appropriate node, otherwise going back to the second step $[29,30]$.

\section{Simulation Results}

Under LoRa wireless communication system, the contradiction of power consumption and real time is well solved, which is of great significance to the development of wireless networks. When using the improved algorithm to optimize wireless communication network, the effect of real time can be optimized to the best. Moreover, the number of nodes is the least. On the one hand, the LoRa wireless network can achieve good communication in a certain area, and, on the other hand, it can meet the requirements of real time and lower power consumption $[8,20,23]$.

In order to show the effect of wireless network nodes optimized by the improved artificial colony algorithm, we select different scene areas to test the two kinds of algorithm. Under the same conditions of external influence, we keep the initial parameters of two kinds of algorithm the same in each scenario. Furthermore, the scene with an area of $100 \backslash 300 \backslash 500 \backslash 700 \backslash 1000 \backslash 1500$ square meters is tested and the power consumption is not limited. In these different areas, the distribution of nodes will have a very intuitive simulation effect, as shown in Table 1.

From Table 1, the improved algorithm is very efficient. Moreover, the proposed algorithm can use fewer nodes to
TABLE 1: Optimization results of the two algorithms in certain scenarios.

\begin{tabular}{lcc}
\hline $\begin{array}{l}\text { Scene area } \\
\left(\mathbf{m}^{2}\right)\end{array}$ & $\begin{array}{c}\text { ABC } \\
(\mathrm{n})\end{array}$ & $\begin{array}{c}\text { RBFABC } \\
(\mathrm{n})\end{array}$ \\
\hline$(100 * 100)$ & 2 & 2 \\
$(300 * 300)$ & 5 & 4 \\
$(500 * 500)$ & 8 & 7 \\
$(700 * 700)$ & 15 & 13 \\
$(1000 * 1000)$ & 24 & 20 \\
$(1500 * 1500)$ & 42 & 32 \\
\hline
\end{tabular}

cover the same area. Above all, the improved algorithm distributes the nodes more evenly in the same space. In a certain area, the distribution of nodes corresponding to the two kinds of algorithm is shown in Figure 2.

Figure 2 shows that, under different areas, the improved algorithm uses fewer nodes. At the same time, the real-time effect of the improved algorithm is better. In general, the improved algorithm obviously has fewer iterations, higher updating speed, and higher convergence speed. What is more, the wireless network realizes the full coverage of the network with fewer nodes by the improved algorithm. Not only that, we also find more advantages of the improved algorithm after deeper analysis of the power consumption. In different scenes, the power consumption of different number of nodes is shown in Figure 3.

Figure 3 shows that the RBFABC algorithm is obviously lower than the $\mathrm{ABC}$ algorithm in terms of power consumption, which shows the superiority of the improved algorithm in wireless network. In most cases, as the area of the scene increases, both the power consumption and the number of nodes also increase. However, we draw a conclusion from Figure 3 that as the number of nodes increases, the power consumption is controlled to a lower level by the improved algorithm. In summary, the RBFABC algorithm solves the contradiction between power consumption and number of nodes better in wireless network.

Correspondingly, to show the superiority of the improved algorithm, a great deal of experiments are done to test the power consumption. For the convenience of data comparison, we still take the scenario area of $100 \backslash 300 \backslash 500 \backslash 700 \backslash 1000 \backslash 1500$ square meters to test. In the case of certain power, the optimized spatial nodes distribution is shown in Figure 4.

It can be concluded from Figure 4 that, under the same power, the RBFABC algorithm is obviously better than the $\mathrm{ABC}$ algorithm in terms of the distribution of the wireless network. Beyond that, fewer nodes cover a wider range by the RBFABC algorithm. To show the superiority of the improved algorithm, we do more tests. Under the same power consumption, the specific number of nodes is shown in Table 2.

According to the data in Table 2, we can draw a conclusion that the RBFABC algorithm is better than the ABC algorithm in terms of optimization effect. Beyond that, the relationship between iteration number and optimal solution is shown in Figure 5. 

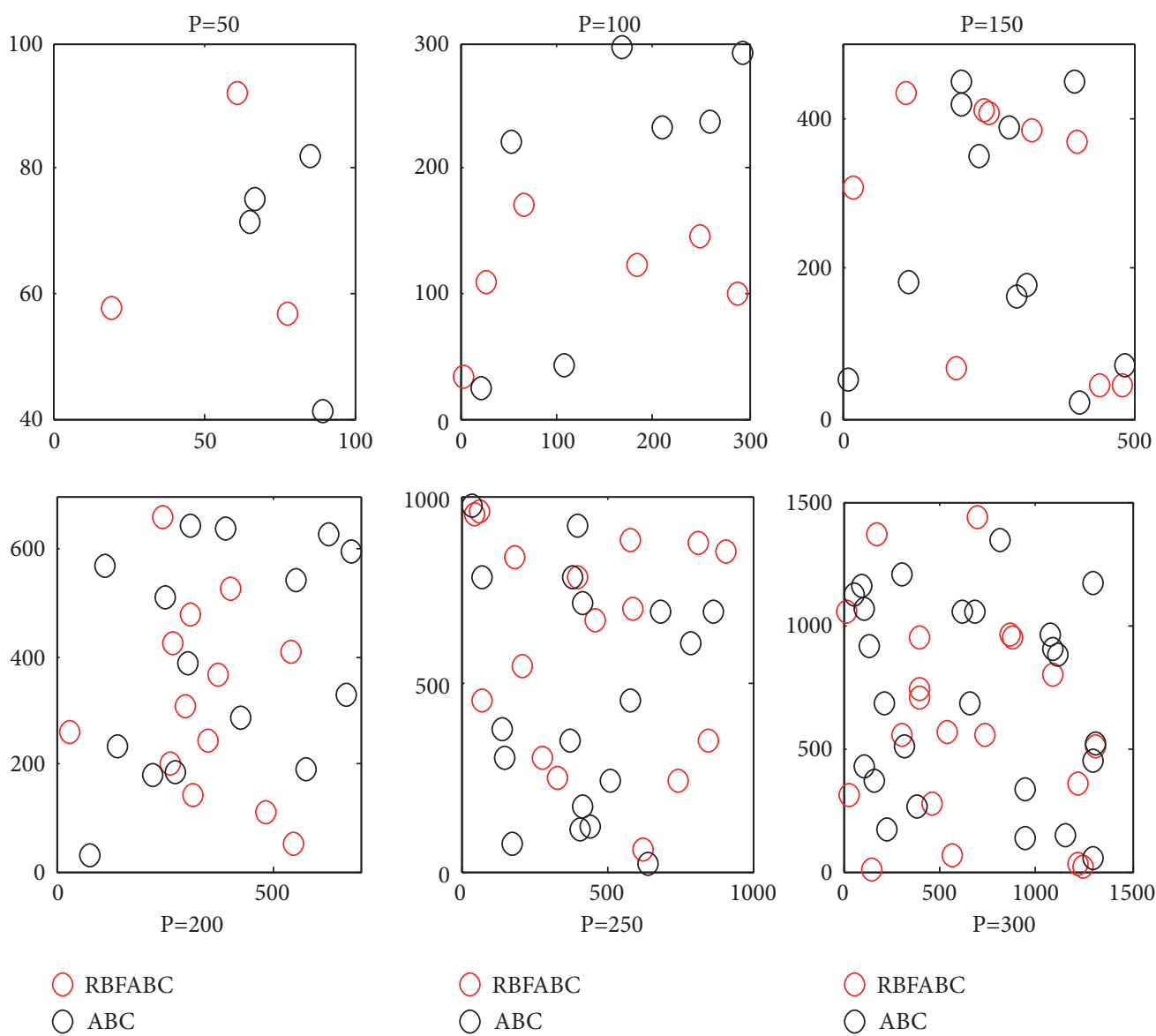

FIGURE 4: Node distribution under the same power.

TABLE 2: Optimization results under a certain power.

\begin{tabular}{lcc}
\hline Power consumption $(\mathrm{w})$ & ABC $(\mathrm{n})$ & RBFABC $(\mathrm{n})$ \\
\hline$(50)$ & 4 & 3 \\
$(100)$ & 7 & 6 \\
$(150)$ & 11 & 9 \\
$(200)$ & 15 & 13 \\
$(250)$ & 18 & 16 \\
$(300)$ & 25 & 20 \\
\hline
\end{tabular}

TABLE 3: Comparison of multimedia data transfer speed.

\begin{tabular}{lcc}
\hline Number of nodes $(\mathrm{n})$ & ABC $(\mathrm{kb} / \mathrm{s})$ & RBFABC $(\mathrm{kb} / \mathrm{s})$ \\
\hline$(5)$ & 200 & 180 \\
$(10)$ & 400 & 350 \\
$(15)$ & 620 & 560 \\
$(20)$ & 810 & 780 \\
$(25)$ & 980 & 850 \\
$(30)$ & 1200 & 990 \\
\hline
\end{tabular}

Figure 5 shows that the improved algorithm can find the optimal solution faster. Therefore, the improved algorithm has more advantages for the optimization of wireless network nodes. Moreover, in a certain area, when the number of network nodes is the same and other factors are consistent, the multimedia data transmission speed which is optimized by two kinds of algorithms is shown in Table 3.

From Table 3, the improved algorithm is obviously superior to the original algorithm in terms of transmission speed. As the number of nodes increases, the data transmission speed becomes faster and faster. In order to display the data visually, the comparison of speed trend is shown in Figure 6.

It is seen from Figure 6 that the RBFABC algorithm is faster than the $\mathrm{ABC}$ algorithm in terms of multimedia data transmission speed. Moreover, as the number of network nodes increases, the data transmission speed gap is bigger and bigger. In general, the RBFABC algorithm is better than the ABC algorithm. Above all, the real-time performance of the system is greatly improved. In addition to these, we do a lot deeper analysis. Under the same number of network nodes, the comparison of network delay is shown in Figure 7.

It can be concluded from Figure 7 that, under the same network nodes, the RBFABC algorithm is obviously lower than the $A B C$ algorithm in terms of network delay. On the other hand, we compare the maximum data load of the single data transmission in the network system. The analysis results of two kinds of algorithm are shown in Figure 8. 


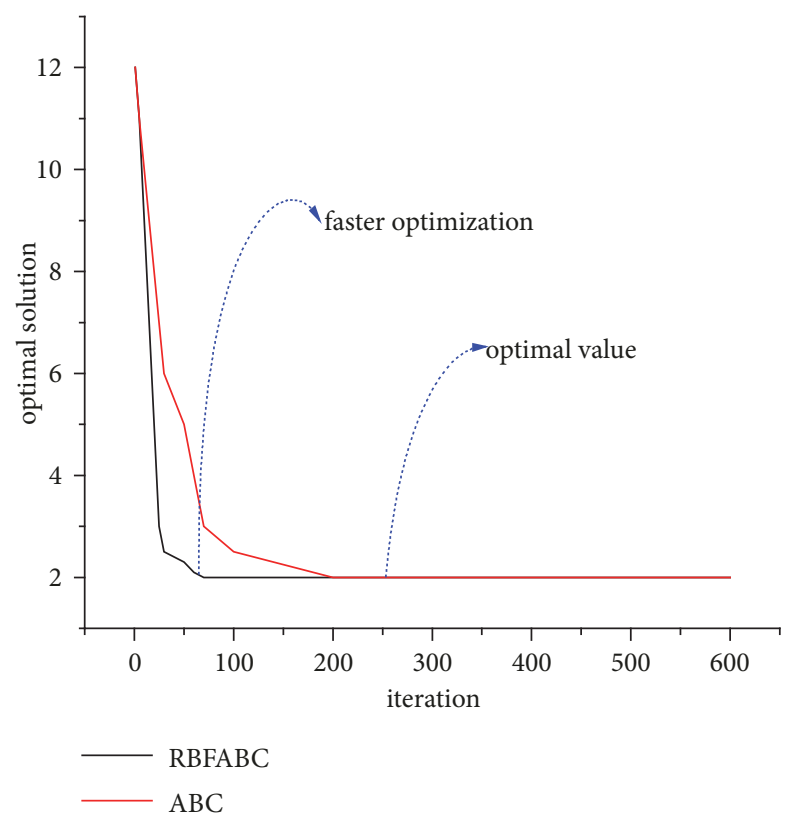

FIgURE 5: The relationship between iteration number and optimal solution.

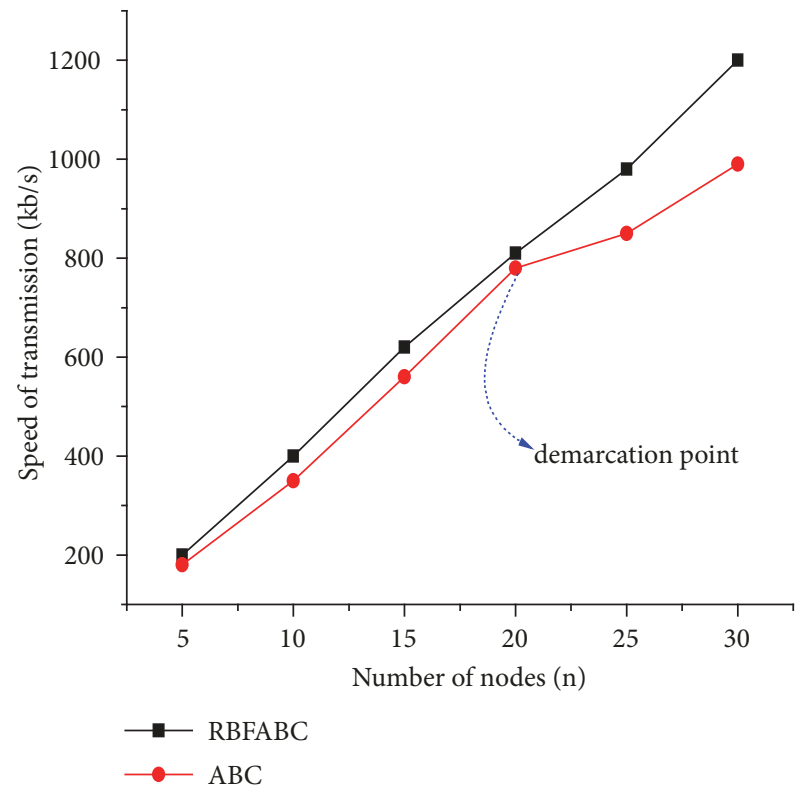

FIGURE 6: Comparison of speed trend.

It is seen from Figure 8 that the RBFABC algorithm is significantly higher than the $\mathrm{ABC}$ algorithm in terms of the maximum data load of the single data interaction. In general, the improved algorithm has more advantages.

\section{Conclusion}

In this paper, to resolve the contradiction of base station establishment in LoRa wireless communication systems

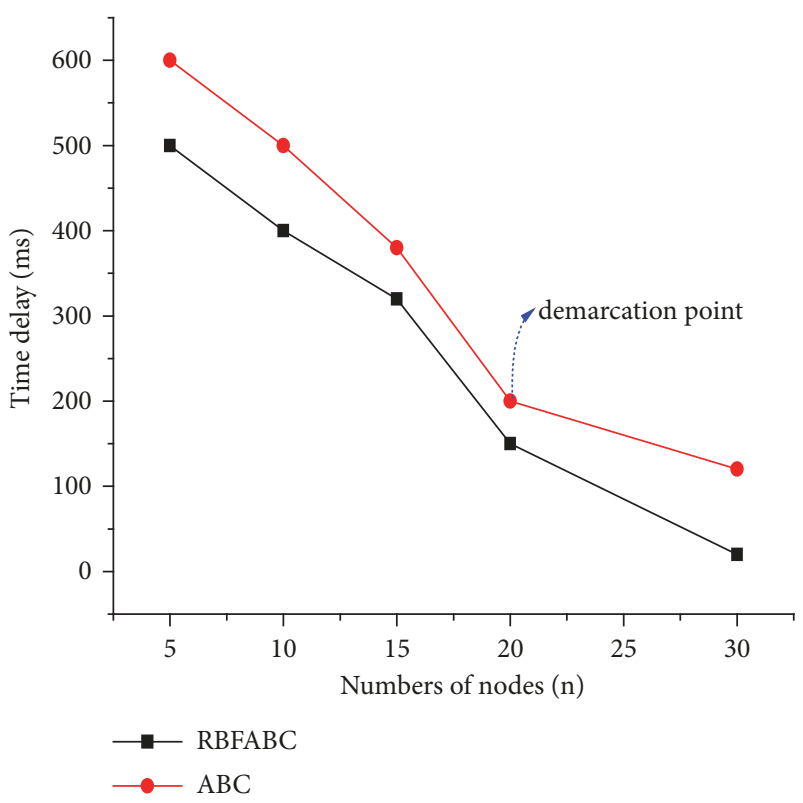

FIGURE 7: Comparison of network delay.

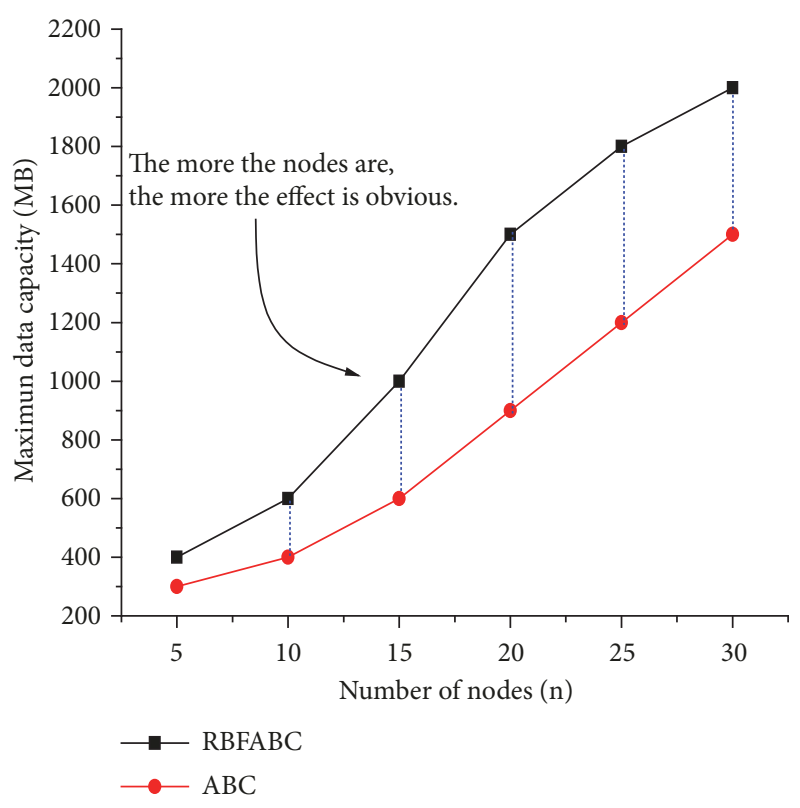

Figure 8: Maximum load comparison.

between the power consumption of LoRa nodes and realtimeliness of the mesh network, we have proposed a novel RBFABC algorithm which achieves better performance by embedding $\mathrm{RBF}$ radial basis neural network training with random gradient method. Extensive simulations have been conducted and the results have shown the obvious superiority of the RBFABC algorithm in terms of the high efficiency and real-timeliness of multimedia transmission. 


\section{Data Availability}

The data used to support the findings of this study are included within the article.

\section{Conflicts of Interest}

The authors declare that they have no conflicts of interest.

\section{Acknowledgments}

This work was supported by Intelligent Manufacturing Project of the Ministry of Industry and Information Technology: Industrial Internet Data Mutual Recognition Research-Low-Power Message Distribution, the National Natural Science Foundation of China under grants 71602124 and 61773187, and Liaoning Provincial Natural Science Foundation of China under grant 20170540662.

\section{References}

[1] K. Wang, H. Yin, W. Quan, and G. Min, "Enabling collaborative edge computing for software defined vehicular networks," IEEE Network, vol. 32, no. 5, pp. 112-117, 2018.

[2] H. Shah, T. Herawan, R. Naseem, and R. Ghazali, "Hybrid Guided Artificial Bee Colony Algorithm for Numerical Function Optimization," in Advances in Swarm Intelligence, vol. 8794 of Lecture Notes in Computer Science, pp. 197-206, Springer International Publishing, Cham, 2014.

[3] Q. Pan, M. F. Tasgetiren, P. N. Suganthan, and T. J. Chua, "A discrete artificial bee colony algorithm for the lot-streaming flow shop scheduling problem," Information Sciences, vol. 181, no. 12, pp. 2455-2468, 2011.

[4] S. kuchlin and P. Jenny, "Automatic mesh refinement and parallel load balancing for Fokker-Planck-DSMC algorithm," Journal of Computational Physics, vol. 363, pp. 140-157, 2018.

[5] X. Ma, S. Xu, F. An, and F. Lin, "A Novel Real-Time Image Restoration Algorithm in Edge Computing," Wireless Communications and Mobile Computing, vol. 2018, Article ID 3610482, pp. 1-13, 2018.

[6] P. Wang and B. Henz, "Efficient approaches to resource allocation in MIMO-based wireless mesh networks," in Proceedings of the 14th Annual Wireless Telecommunications Symposium, WTS '15, vol. 39, pp. 1-7, USA, April 2015.

[7] F. Lin and X. Lü, "QoS guaranteed pre-pushing scheme in peerassisted streaming network," China Communications, vol. 11, no. 14, pp. 111-117, 2014.

[8] J. Wellons and Y. Xue, "The robust joint solution for channel assignment and routing for wireless mesh networks with time partitioning," Ad Hoc Networks, vol. 13, pp. 210-221, 2014.

[9] H. Chaoui, M. Khayamy, and O. O. Okoye, "Adaptive RBF Network Based Direct Voltage Control for Interior PMSM Based Vehicles," IEEE Transactions on Vehicular Technology, 2018.

[10] W. Zhuang and M. Ismail, "Cooperation in wireless communication networks," IEEE Wireless Communications Magazine, vol. 19, no. 2, pp. 10-20, 2012.

[11] J. Si, H. Huang, Z. Li, B. Hao, and R. Gao, "Performance analysis of adaptive modulation in cognitive relay network with cooperative spectrum sensing," IEEE Transactions on Communications, vol. 62, no. 7, pp. 2198-2211, 2014.
[12] M. Benedetto, K. Johansson, and M. Johansson, "Industrial control over wireless networks," International Journal of Robust \& Nonlinear Control, vol. 20, no. 2, pp. 119-127, 2018.

[13] C. M. Stefanovic, "LCR of amplify and forward wireless relay systems in general alpha-Mu fading environment," in Proceedings of the 2017 25th Telecommunication Forum (TELFOR), pp. 1-6, Belgrade, November 2017.

[14] D. Prasad, V. Koneri, and K. Shivakumar, "Mitigating wireless network interface card energy consumption in mobile devices," Research \& Technology in the Coming Decades, vol. 2014, no. 1, pp. 103-114, 2014.

[15] E. Aivaloglou and S. Gritzalis, "Hybrid trust and reputation management for sensor networks," Wireless Networks, vol. 16, no. 5, pp. 1493-1510, 2010.

[16] J. Wu, X. Qiao, Y. Xia, C. Yuen, and J. Chen, "A low-latency scheduling approach for high-definition video streaming in a heterogeneous wireless network with multihomed clients," Multimedia Systems, vol. 21, no. 4, pp. 411-425, 2015.

[17] M. Ghaderi, D. Goeckel, A. Orda, and M. Dehghan, "Minimum Energy Routing and Jamming to Thwart Wireless Network Eavesdroppers," IEEE Transactions on Mobile Computing, vol. 14, no. 7, pp. 1433-1448, 2015.

[18] S. Dasgupta, G. Mao, and B. Anderson, "A New Measure of Wireless Network Connectivity," IEEE Transactions on Mobile Computing, vol. 14, no. 9, pp. 1765-1779, 2015.

[19] X. Cheng, Q. Wang, Q. Wang, and D. Wang, "A high-reliability relay algorithm based on network coding in multi-hop wireless networks," Wireless Networks, pp. 1-10, 2017.

[20] Y. E. Sagduyu, Y. Shi, A. Fanous, and J. H. Li, "Wireless network inference and optimization: Algorithm design and implementation," IEEE Transactions on Mobile Computing, vol. 16, no. 1, pp. 257-267, 2017.

[21] J. Liu and X. Wu, "Supervision and control based on rbf neural network," Computer Knowledge Technology, vol. 2018, pp. 1-13, 2018.

[22] H. Qin, L. Shen, C. Sima, and Q. Ma, "RBF Networks with Dynamic Barycenter Averaging Kernel for Time Series Classification," in Artificial Intelligence, vol. 888 of Communications in Computer and Information Science, pp. 139-152, Springer Singapore, Singapore, 2018.

[23] P. Wang, "The Application of Radial Basis Function (RBF) Neural Network for Mechanical Fault Diagnosis of Gearbox," IOP Conference Series: Materials Science and Engineering, vol. 269, pp. 012-056, 2017.

[24] J. Dong and L. Chen, "The nonlinear integral sliding mode of RBF neural network algorithm is used to control the motion trajectory error of the manipulator," Chinese Journal of Construction Machinery, vol. 2018, no. 17, pp. 11-22, 2018.

[25] Y. Ma and C. Na, "Switch fault diagnosis system based on cost sensitive RBF neural network," Railway Computer Application, vol. 2018, no. 13, pp. 45-53, 2018.

[26] H. Du, E. Zhao, K. Guo, and H. Zhing, "Safety monitoring model of dam service based on genetic algorithm and RBF neural network," Journal of China Three Gorges University, vol. 2018, no. 18, pp. 15-27, 2018.

[27] D. Zaborski, W. Grzesiak, and M. Szewczuk, "An application of radial basis function (RBF) networks to daily body weight gains prediction in the indigenous Harnai Sheep of Pakistan," Publication Preview Source Biotechnologia Problemy Wyzwania Sczecin Poland, vol. 14, no. 18, pp. 67-87, 2018. 
[28] D. Karaboga and B. Akay, "A modified Artificial Bee Colony (ABC) algorithm for constrained optimization problems," Applied Soft Computing, vol. 11, no. 3, pp. 3021-3031, 2011.

[29] D. Karaboga, B. Gorkemli, C. Ozturk, and N. Karaboga, "A comprehensive survey: artificial bee colony $(\mathrm{ABC})$ algorithm and applications," Artificial Intelligence Review, vol. 42, pp. 2157, 2014.

[30] J.-J. Forneron and S. Ng, "The ABC of simulation estimation with auxiliary statistics," Journal of Econometrics, vol. 205, no. 1, pp. 112-139, 2018. 


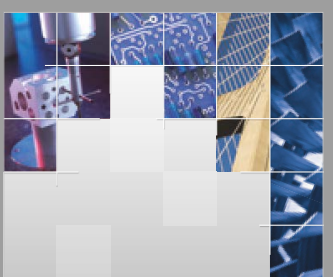

\section{Enfincering}
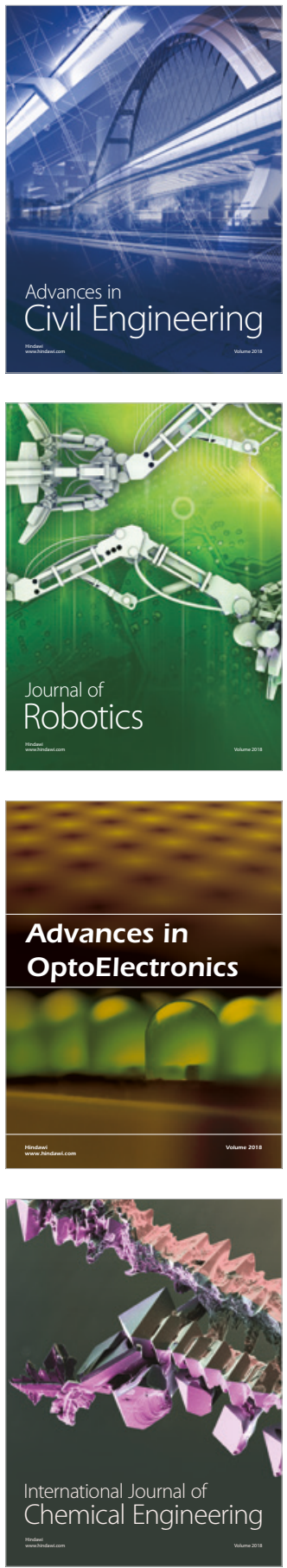

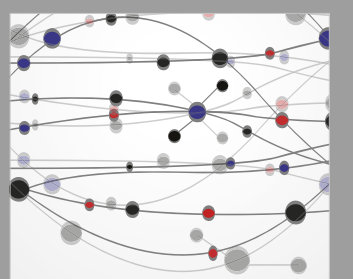

\section{Rotating \\ Machinery}

The Scientific World Journal

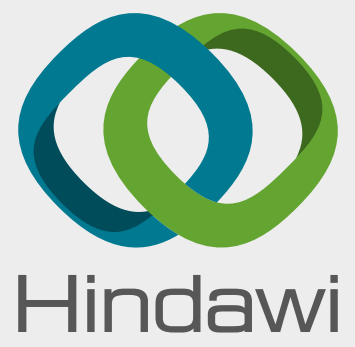

Submit your manuscripts at

www.hindawi.com
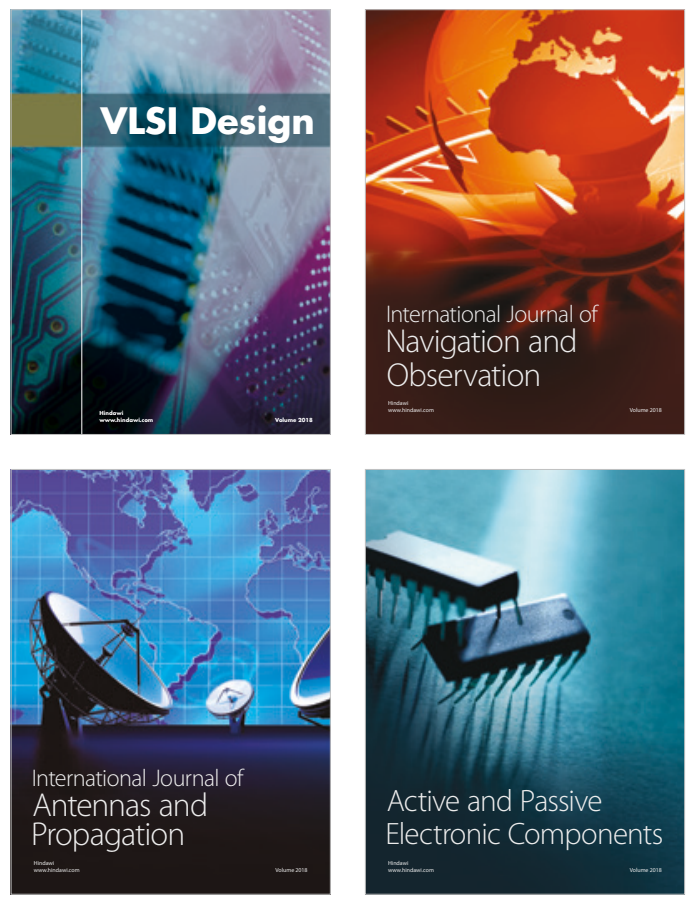
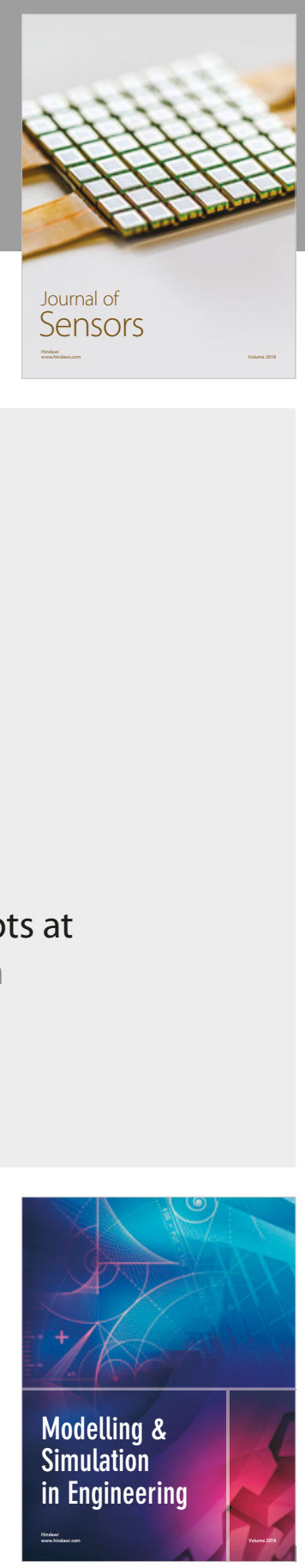

\section{Advances \\ Multimedia}
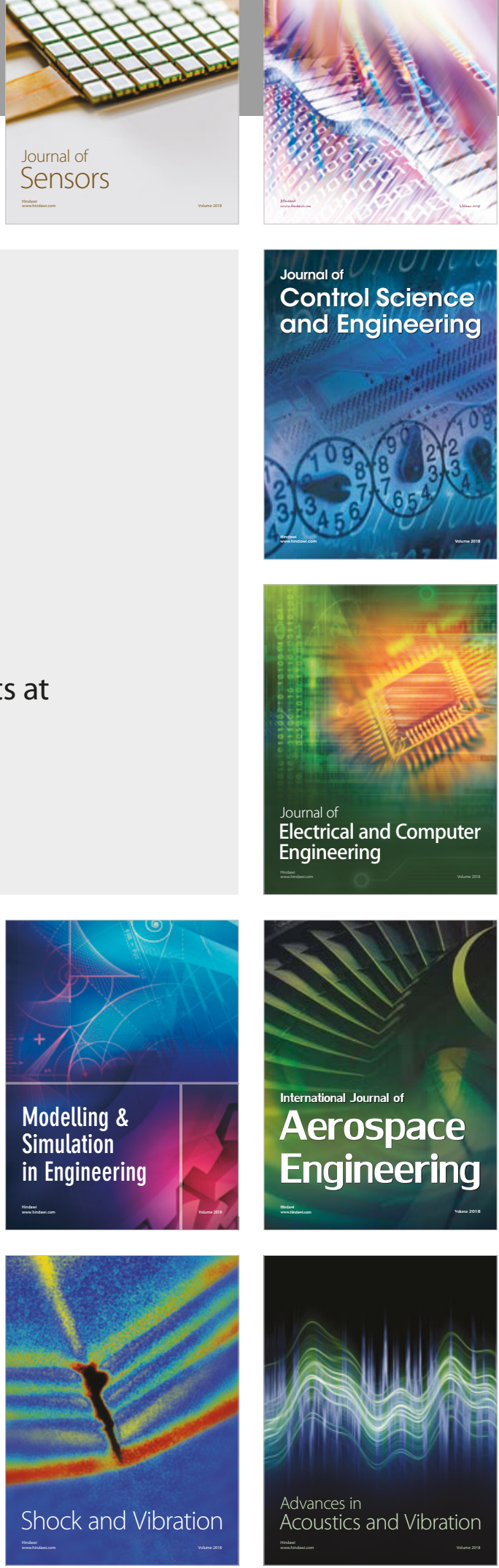